\title{
$\mathrm{ICONO} 14$
}

\section{TikTok y Twitch: nuevos medios y fórmulas para impactar en la Generación Z}

\section{TikTok y Twitch: new media and formulas to impact the Generation Z}

\section{TikTok e Twitch: novas mídias e fórmulas para impactar a Geração Z}

Ainhoa García Rivero ${ }^{1}$ D

Eva Citlali Martínez Estrella²

Gema Bonales Daimiel $\left.\right|^{3}$ iD

${ }^{1}$ Investigadora (Universidad Complutense de Madrid), España

${ }^{2}$ Investigadora (Universidad Complutense de Madrid), España

${ }^{3}$ Profesora Ayudante Doctor (Universidad Complutense de Madrid), España

Recibido: 30/06/2021; Revisado: 14/07/2021; Aceptado: 04/11/2021; Publicado: 01/01/2022

Para citar este artículo: García Rivero, A., Citlali Martínez Estrella, E. y Bonales Daimiel, G. (2022). TikTok y Twitch: nuevos medios y fórmulas para impactar en la Generación Z, Icono 14, 20(1). https://doi.org/10.7195/ri14.v20i1.1770

\section{Resumen}

El desinterés de los jóvenes por los medios tradicionales y su sustitución por plataformas de vídeo bajo demanda y redes sociales ha provocado que TikTok y Twitch se conviertan en las principales fuentes de información y entretenimiento para ellos, exigiendo una evolución en los contenidos de las marcas (IAB, 2021).

El objetivo de esta investigación es conocer la percepción que tiene la Generación Z en España sobre la comunicación comercial de las marcas con mayor presencia en TikTok y Twitch, así como establecer su nivel de reconocimiento. Asimismo, se determina la 
usabilidad de estas redes y qué acciones comunicativas son las más aceptadas por este segmento de edad.

Para ello se ha realizado una técnica mixta, basada principalmente en la realización de un focus group a ocho jóvenes, cinco entrevistas a expertos y una encuesta $(n=420)$. La principal conclusión es que a la industria publicitaria le falta transformar sus estrategias de comunicación dirigidas a la Generación Z. También, los jóvenes recuerdan más las marcas que aparecen como patrocinadoras en eventos de Twitch; además, las marcas son mejor valoradas en Twitch que en TikTok. Respecto a la usabilidad, estas plataformas ya no solo proporcionan entretenimiento, sino que se han convertido en herramientas para estudiar y en medios de socialización.

Palabras clave: Generación Z; TikTok; Twitch; comunicación comercial; patrocinios; publicidad

\begin{abstract}
The loss of interest of young people in traditional media and their replacement by video-ondemand platforms and social networks has led TikTok and Twitch to become the main sources of information and entertainment for them, demanding an evolution in the content of brands (IAB, 2021).

The aim of this research is to know the perception that Generation Z in Spain has about the commercial communication of brands with higher presence in TikTok and Twitch, as well as to establish their level of recognition. It also determines the usability of these networks and which communicative actions are the most accepted by this age segment.
\end{abstract}

For this purpose, a mixed technique was used, based mainly on a focus group of eight young people, five interviews with experts and a survey $(n=420)$. The main conclusion is that the advertising industry is lacking in transforming its communication strategies aimed at Generation Z. Also, young people remember more brands that appear as sponsors in Twitch events; moreover, brands are better valued on Twitch than on TikTok. Regarding usability, these platforms no longer only provide entertainment, but have become tools for studying and socializing.

Keywords: Generation Z; TikTok; Twitch; commercial communication; sponsorships; advertising

\title{
Resumo
}

O desinteresse dos jovens pelos meios de comunicação tradicionais e a sua substituição por plataformas de vídeo a pedido e redes sociais levou a que o TikTok e o Twitch se tornassem as principais fontes de informação e entretenimento para eles, exigindo uma evolução no conteúdo das marcas (IAB, 2021).

O objectivo desta investigação é conhecer a percepção que a Geração Z em Espanha tem sobre a comunicação comercial das marcas com maior presença em TikTok e Twitch, bem como estabelecer o seu nível de reconhecimento. Também determina a usabilidade destas redes e quais as ações comunicativas que são mais aceites por este segmento etário. 
Para este fim, foi utilizada uma técnica mista, baseada principalmente num grupo focal de oito jovens, cinco entrevistas com peritos e um inquérito $(n=420)$. A principal conclusão é que a indústria publicitária não consegue transformar as suas estratégias de comunicação destinadas à Geração Z. Além disso, os jovens lembram-se mais das marcas que aparecem como patrocinadores nos eventos Twitch; além disso, as marcas são mais valorizadas no Twitch do que no TikTok. Quanto à usabilidade, estas plataformas já não fornecem apenas entretenimento, mas tornaram-se instrumentos de estudo e de socialização.

Palavras-chave: Geração Z; TikTok; Twitch; comunicação comercial; patrocínios; publicidade

\section{Introducción}

El auge de la comunicación digital y, en concreto, las redes sociales, han transformado los hábitos de consumo (Martínez, García y Gutiérrez, 2013). Una de las consecuencias es que las plataformas de vídeo bajo demanda ahora sean las más utilizadas como fuente de información y de entretenimiento (IAB, 2021; Suárez-Álvarez y García-Jiménez, 2021). Consecuentemente, el uso de estas hace que la percepción que tienen los públicos de las empresas y de su comunicación cambie, ocasionando que los usuarios se apropien de los contenidos que más les gustan y generen nuevos mensajes a partir de las publicaciones de las marcas (Mir, 2016; Keller y Swaminathan, 2019; Lacasa, 2018; Tur-Viñes et al., 2018). Los usuarios tienen el poder de decidir si consumen o no lo que aparece en sus perfiles de redes sociales, por lo que la innovación y creatividad en los nuevos formatos de comunicación se convierten en elementos indispensables (Karbaum, 2018; Toffler, 1980).

TikTok y Twitch, son consideradas las nuevas redes sociales y están dirigidas principalmente a un público joven -entre 16 y 24 años- (Epsilon Technologies, 2020). Además, la Generación Z muestra "una clara preferencia por lo visual en los medios sociales" (Álvarez, Heredia y Romero, 2019, p. 2), y esa es una de las principales características de las plataformas que se analizan en este trabajo.

El objetivo de esta investigación es contrastar la visión que tiene la industria publicitaria al diseñar y planificar anuncios en estas redes sociales, siguiendo la percepción que posee la Generación Z en España sobre la comunicación comercial de las marcas. Asimismo, se pretende determinar la usabilidad de estas redes destacando si existe una dependencia entre sexo y el tipo de contenido que se consume, al igual que identificar qué acciones comunicativas son las más aceptadas por este segmento de edad en dichas plataformas. 


\subsection{Comunicación comercial en redes sociales}

Las redes sociales son espacios en Internet donde personas y organizaciones mantienen una comunicación activa y se interrelacionan (Mir, 2016). Los consumidores, entre otras cosas, comparten información en línea, dejando de ser espectadores para convertirse en productores de contenido, siendo su comportamiento independiente de las acciones que implementan las marcas (Pedroni, 2013). Fernández de la Iglesia, Casal, Fernández y Cebreiro (2020) hacen referencia a un elevado uso de redes sociales por parte de los jóvenes, porque Internet se ha convertido en un sitio de ocio y entretenimiento (GarcíaJiménez et al., 2018), así como "un lugar no solo para mantener las amistades, sino para entablar otras nuevas" (Martínez, García y Sendín, 2013, p. 120).

Según Soler (2016), los adolescentes encuentran actitudes de confianza tanto en los agentes sociales (familia, escuela...) como en las redes sociales, evidenciando una evolución en las prácticas sociales a través de la comunicación en dichas plataformas. Para los jóvenes, las redes sociales son espacios de socialización donde crean su identidad y se comunican con sus iguales y otros miembros de la comunidad virtual (Segovia, Mérida, Olivares y González, 2016; Álvarez, Heredia y Romero, 2019). Así, "las TIC se han convertido en un nuevo contexto de relaciones interpersonales entre los jóvenes" (Espinar, Zych y Rodríguez-Hidalgo, 2015).

Sin embargo, la publicidad digital es percibida por muchos usuarios (especialmente por el grupo de edad que nos ocupa) como algo molesto que les hace perder velocidad de navegación y/o impide acceder a los contenidos, especialmente en los dispositivos móviles (Martínez-Costa, Serrano-Puche, Portilla, Sánchez-Blanco, 2019). Gálvez (2017) señala que el 59\% de los internautas mayores de 14 años conocen los adblockers (bloqueadores de anuncios) y que un $28 \%$ los utiliza regularmente.

En este contexto, es fundamental hablar de la prescripción en los jóvenes. Tal y como señala Varona (2013), la opinión y prescripción de los amigos es un aspecto fundamental a tener en cuenta cuando se trabaja con adolescentes. A los jóvenes, según este, les resulta más creíble y sincera la opinión de un amigo sobre una determinada marca que el propio mensaje que la marca pueda transmitirle, algo que también comparten Cooley y ParksYancy (2019). Aquí cobra especial relevancia el hecho de que las marcas confíen la difusión de su mensaje a los streamers. Elías (2021) asegura que esta generación: "busca en los streamers de Twitch una 'relación' a largo plazo".

Al respecto, Ferrer (2020) agrega que los contenidos protagonizados por influencersson los que mayores picos de atención generan entre el público joven, al igual que afirma que los contenidos cuya publicidad es más encubierta, como el branded content, Ilaman más la atención a los jóvenes que los spots publicitarios protagonizados por un influencer. Estas acciones, según Castelló y del Pino (2015), deben "entenderse como parte de la estrategia de comunicación integral de la marca en una época en la que la publicidad ha de ser transmedia y multidisciplinar". 


\subsection{Generación Z, TikTok y Twitch}

La realidad es que "no existe consenso definitivo en cuanto a fechas de inicio y fin para nombrar a las generaciones más jóvenes" (Marinas, 2019; Francisco y Rodríguez, 2020). Para esta investigación se consideran los jóvenes nacidos entre el 2000 y 2007 (de 14 a 21 años), quienes son los principales usuarios de las redes sociales seleccionadas para el análisis.

Thomas (2020) describe a esta generación como digital y con mentalidad social; la define como la más diversa. Mientras que Castelló-Martínez (2020) destaca la importancia de los valores corporativos y la responsabilidad social de las marcas en su comunicación cuando se dirigen a este target.

Según Martín y Medina (2021), “los miembros de la generación Z dedican más de 3 horas diarias a las redes sociales" (p. 72). Por su parte, Álvarez, Heredia y Romero (2019) señalan que los jóvenes son expertos en el uso de las TIC al haber accedido a ellas de manera autodidacta. Vilanova y Ortega (2017) consideran que Internet es uno de los elementos que definen a esta generación, junto con la irreverencia, la inmediatez y la incertidumbre.

La inmediatez e impaciencia que les caracteriza ha provocado que se alejen paulatinamente de la televisión para migrar hacia otro tipo de plataformas VOD (video on demand) como "televisión tradicional a la carta, plataformas como Netflix o HBO o plataformas de vídeo como YouTube o Twitch" (Gutiérrez y Cuartero, 2020, p. 167), un hecho al que otros autores también hacen referencia (Navarro y Vázquez, 2020; Marcos et al., 2020). Además: "los jóvenes son los usuarios que mejor valoran los contenidos audiovisuales online debido a motivos como la instantaneidad espacio-temporal, la variedad de contenidos o la identificación con los creadores de los vídeos" (Navarro y Vázquez, 2020, p. 11). Francisco y Rodríguez (2020), aseguran que los miembros de esta generación hacen un uso "complementario de los dos medios" (Internet y televisión) la gran mayoría de las veces (p. 193).

En cuanto a las redes elegidas para esta investigación, Twitch era inicialmente una plataforma donde los jugadores de videojuegos transmitían en directo sus partidas. No obstante, a raíz de la pandemia provocada por la Covid-19, esta red ha experimentado un aumento masivo en su uso, especialmente en categorías que no se relacionan con los videojuegos. Una de las características de Twitch que ha hecho que su popularidad crezca es la posibilidad de interacción con la audiencia en directo a través del chat (Wilson, 2021; Gutiérrez y Cuartero, 2020).

Tal y como se puntualiza en el propio sitio web, "Twitch está forjando el futuro del entretenimiento interactivo en vivo" (Twitch, 2021). En cuanto a su audiencia, gran parte de ella pertenece a la Generación Z; de acuerdo con el estudio realizado por Wilson para 
Harvard Business Review, "el 21\% tiene entre 13 y 17 años y casi la mitad entre 18 y 34 años" (2021).

Gutiérrez y Cuartero refieren a "la paradoja de Twitch" (2020, p. 168) señalando que se puede observar cómo diferentes figuras provenientes de los medios tradicionales (como es la televisión) pueden trasladar "su audiencia desde estas plataformas más consolidadas hacia nuevas ventanas como Twitch, gracias sobre todo a la capacidad de estos creadores de contenidos para conectar con el público juvenil" (p. 169).

Por otro lado, TikTok es una red social cuyos usuarios mayoritariamente también corresponden a la Generación Z (Li, Xiaohui y Zhengwu, 2019; Shuai et al., 2019; Wilson, 2021) aunque durante el 2020, debido a la pandemia de la Covid-19, se reportó un incremento de usuarios Millennials y de la Generación X. Su formato es un vídeo en vertical y su contenido es principalmente humorístico, y de playback, (Chaoudhary, Gautam y Arya, 2020).

TikTok demanda rapidez de producción y consumo, lo que se traduce en una exigencia creativa y, en la mayoría de las ocasiones, vinculada con música (Li, Xiaohui y Zhengwu, 2019). El contenido de marca en la plataforma es, a menudo, indistinguible del que es creado por los propios usuarios (Wilson, 2021).

En definitiva, se puede considerar que Twitch y TikTok ponen en evidencia un cambio de tendencia en cuanto al consumo audiovisual de los jóvenes y "provocan una transformación más amplia en la forma en la que esta audiencia se relaciona con un producto audiovisual" (Gutiérrez y Cuartero, 2020, p. 171).

\subsection{Objetivos}

El objetivo general de esta investigación es contrastar la visión de la industria publicitaria sobre la comunicación en TikTok y Twitch con la percepción que tiene la Generación Z de ella y sobre las marcas que la realizan.

Del mismo modo, como objetivos específicos, se pretende identificar el contenido más consumido en ambas plataformas y la usabilidad de las mismas, así como conocer si este consumo tiene una relación de dependencia con el sexo del usuario. Adicionalmente, también se pretende evaluar el reconocimiento que tiene la audiencia en estas redes sociales sobre las marcas anunciantes e identificar las acciones comunicativas y publicitarias con mayor aceptación por parte de los usuarios de la Generación Z. 


\section{Material y Métodos}

La metodología aplicada tiene un enfoque mixto con un carácter empírico analítico (Batthyány y Cabrera, 2011), porque a través de la interpretación de los datos se obtienen las principales conclusiones. Al no contar con una muestra representativa, se diseñó un estudio exploratorio, considerando a la población joven española (nacidos entre el 2000 y 2007).

La investigación comienza con una revisión bibliográfica, seguida por un focus group sobre la usabilidad en ambas plataformas: a raíz de esta información, se elaboran las entrevistas a usuarios y expertos. Finalmente, con todos los datos cualitativos, se realiza el cuestionario para confirmar las opiniones de los expertos y diseñar los patrones de consumo en Twitch y TikTok. Por tanto, la investigación se divide en tres fases: 1) acercamiento a la Generación Z; 2) visión de la industria publicitaria; y 3) encuesta de exploración.

En la primera fase, se hizo un grupo de discusión para examinar las perspectivas e intereses de los usuarios en Twitch y TikTok. Se contó con un grupo de 8 adolescentes de entre 15 y 19 años ya que, de acuerdo con la revisión teórica, los principales usuarios de Twitch pertenecen a esta franja de edad (Gutiérrez y Cuartero, 2020; Blanco, 2020). Como requisitos de participación, además de encontrarse en el rango de edad seleccionado, los participantes tenían que utilizar Twitch y TikTok habitualmente.

Posteriormente, en la segunda fase se desarrollaron entrevistas en profundidad a cuatro profesionales de la industria para hablar sobre las estrategias de comunicación de las marcas en estas redes sociales cuyo target es la Generación Z. Los entrevistados fueron Olga Diez y Katty Huerta (CEO y Market Research Analyst en The Onion Inside, respectivamente), Gaby Rabinowicz (Co-Founder de Kidsmedia) y Álvaro de la Cruz (Director de estrategia en The Modern Kids and Family).

Al igual que las entrevistas, el focus group fue realizado online por los miembros del equipo investigador durante marzo y mayo de 2021.

En la tercera fase, se elaboró un cuestionario (basado en la información obtenida en las técnicas anteriores). El universo de la muestra fue de mujeres y hombres españoles de 14 a 21 años que fuesen usuarios activos de TikTok y Twitch. La selección de los participantes fue aleatoria y se consiguieron 420 respuestas. El cuestionario online estuvo en circulación del 18 de marzo al 18 de abril de 2021.

La encuesta tuvo un desarrollo temático con respuestas semiestructuradas divididas en cuatro apartados:

1)Datos sociodemográficos 
Usabilidad y consumo en TikTok/Twitch

Percepción de marcas en TikTok /Twitch

Publicidad ideal en TikTok /Twitch

Con este cuestionario se pretendía conocer la experiencia del usuario en cada red, su percepción sobre las marcas anunciadas en estas redes sociales e identificar los contenidos que más consumen en cada plataforma.

Asimismo, se solicitó a los encuestados que valorasen la comunicación de las marcas con más presencia en estas redes sociales según los rankings elegidos. Las valoraciones de marcas se realizaron a través de una escala de Likert de cinco opciones: muy buena, buena, regular, mala y muy mala; considerando las tres primeras como consideración "buena" y las dos últimas como consideración "mala". Adicionalmente, existía la opción NS/NC (no sabe/no contesta).

Para determinar el reconocimiento de las marcas y la valoración de estas en ambas plataformas, se utilizaron dos rankings que presentan las marcas con mayor visibilidad, interacción y publicidad pagada en TikTok y YouTube en España en 2020. Al no existir un índice de marcas que fuera exclusivo de Twitch, se utilizó el que correspondía a YouTube debido a que esta red social es la predecesora de Twitch (Scully-Blaker, Begy, Consalvo y Ganzon, 2017) y a que son plataformas interconectadas, como posteriormente se demostrará en los resultados. Además, cabe destacar que estas dos redes sociales: YouTube y Twitch, se estudian conjuntamente con frecuencia (Pires y Simon, 2015; Sidhu y Carter, 2020; Pollack et al., 2021; Jung y Phoa, 2021).

En el caso de TikTok, se eligió el informe Tendencias y Rankings de top marcas en TikTok España elaborado por la agencia de big dataEpsilon Technologies (2020), en el que se establecía que el Top 10 de marcas en TikTok España según la cuota de mercado de interacciones estaba conformado de la siguiente manera: Red Bull, LaLiga, Movistar+, Telecinco, El Chiringuito de Jugones, Cosmopolitan, As, Cruz Roja, Sport y Mundo Deportivo. Para elegir las marcas de Twitch se empleó el ranking elaborado por la agencia de medios digitales 2btube (2020), donde Red Bull es el líder del Top 10 de YouTube, seguido por BBVA, Playstation España, Fornite, Ubisoft, Myprotein, Vodafone, Nintendo, L'Oréal y Sports FIFA.

Por último, se hizo una pregunta abierta para conocer las características que los usuarios consideran fundamentales para que la publicidad no les resultase molesta.

Una vez obtenidos todos los datos, se realizó un análisis temático con el programa Atlas.ti. El primer paso fue resumir y contrastar la información del focus group y de las entrevistas, elaborando mapas conceptuales que muestran las relaciones y las dependencias temáticas entre los usuarios y los expertos. Esta separación fue útil para establecer los patrones de consumo y la relevancia de TikTok y Twitch en la comunicación comercial. Después, se valoró el patrón de consumo de estas plataformas y se aplicó la fórmula estadística de chi- 
cuadrado para confirmar si el sexo determina el tipo de contenido consumido, teniendo un margen de error del 5\% y un grado de confianza del 95\%. Las variables empleadas fueron el sexo (hombre o mujer) y la tipología de contenidos de ambas plataformas (Tabla 1).

Tabla 1. Tipología de contenido

\begin{tabular}{|l|l|}
\hline Tipología en Twitch & Tipología en TikTok \\
\hline Partidas de videojuegos & Tips de estudio, decoración y cocina \\
\hline Eventos deportivos & Rutinas diarias \\
\hline Entrevistas & Videos cómicos \\
\hline Eventos musicales & Challenges \\
\hline Reacciones de vídeos de YouTube & Material de marcas e influencers \\
\hline Canal de lbai & Noticias, temas polémicos \\
\hline Grupos de estudio & \\
\hline
\end{tabular}

Fuente: Elaboración propia

\section{Resultados}

\subsection{Focus Group}

En el Gráfico 1 se observan las relaciones existentes entre las cinco categorías principales de la investigación. Se puede resaltar que durante toda la sesión los participantes se mostraron entusiastas y con predisposición a compartir su experiencia a través de ejemplos de vivencias y opiniones interesantes sobre estas redes sociales, así como haciendo mención a cuáles son sus contenidos y streamers favoritos. 


\section{Gráfico 1. Categorías temáticas del focus group}

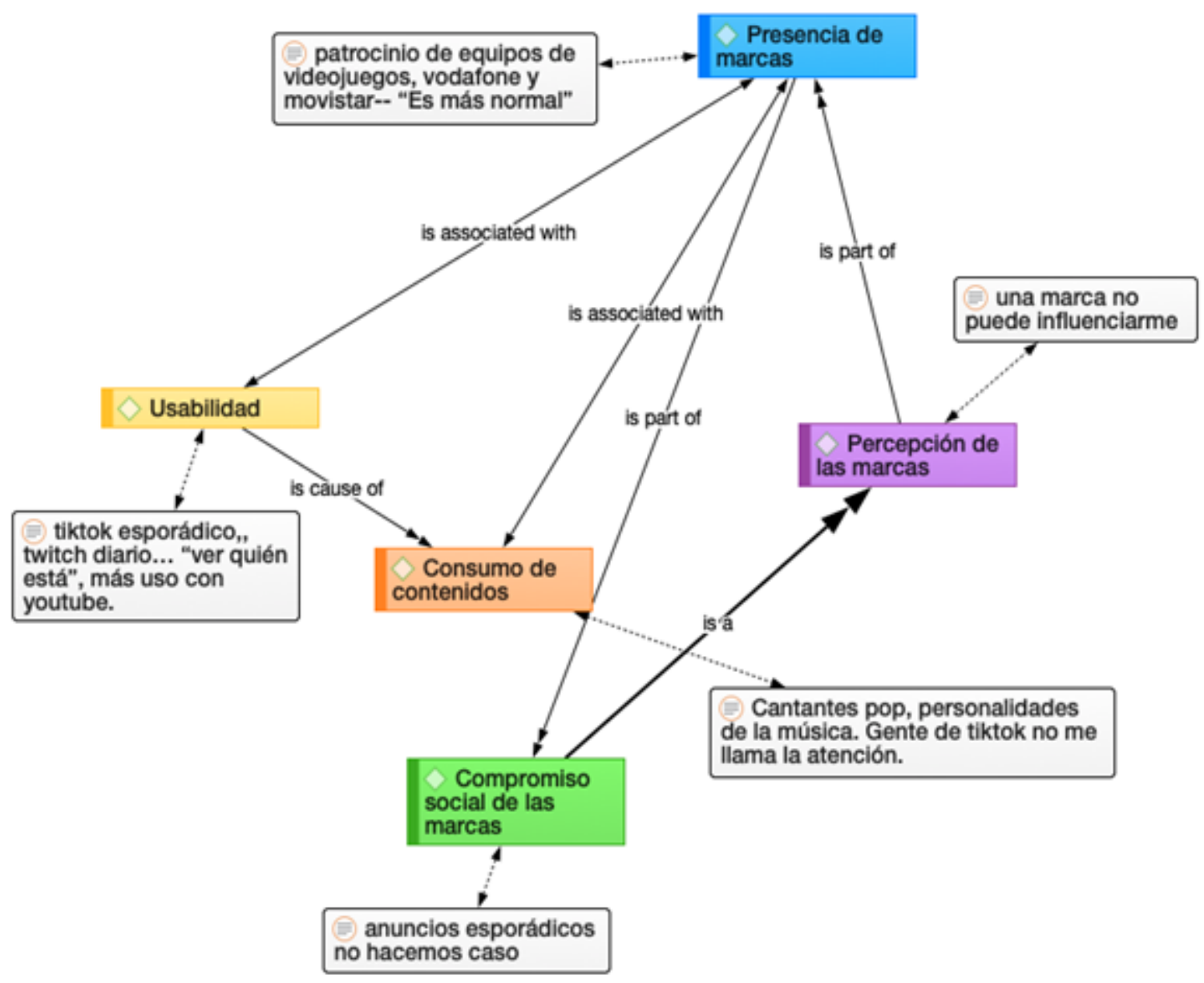

Fuente: Elaboración propia

\subsection{Entrevistas en profundidad}

El anterior focus group se complementa con las entrevistas a expertos. La principal conclusión obtenida de estas entrevistas es que no todas las marcas pueden tener presencia en Twitch, ya que sus peculiaridades de usabilidad hacen que el principal portavoz de los mensajes sean los streamers y no las marcas directamente. Igualmente, los profesionales coinciden en que es una red social que representa un mayor coste para la marca. 
Gráfico 2. Resultados de entrevistas a profesionales

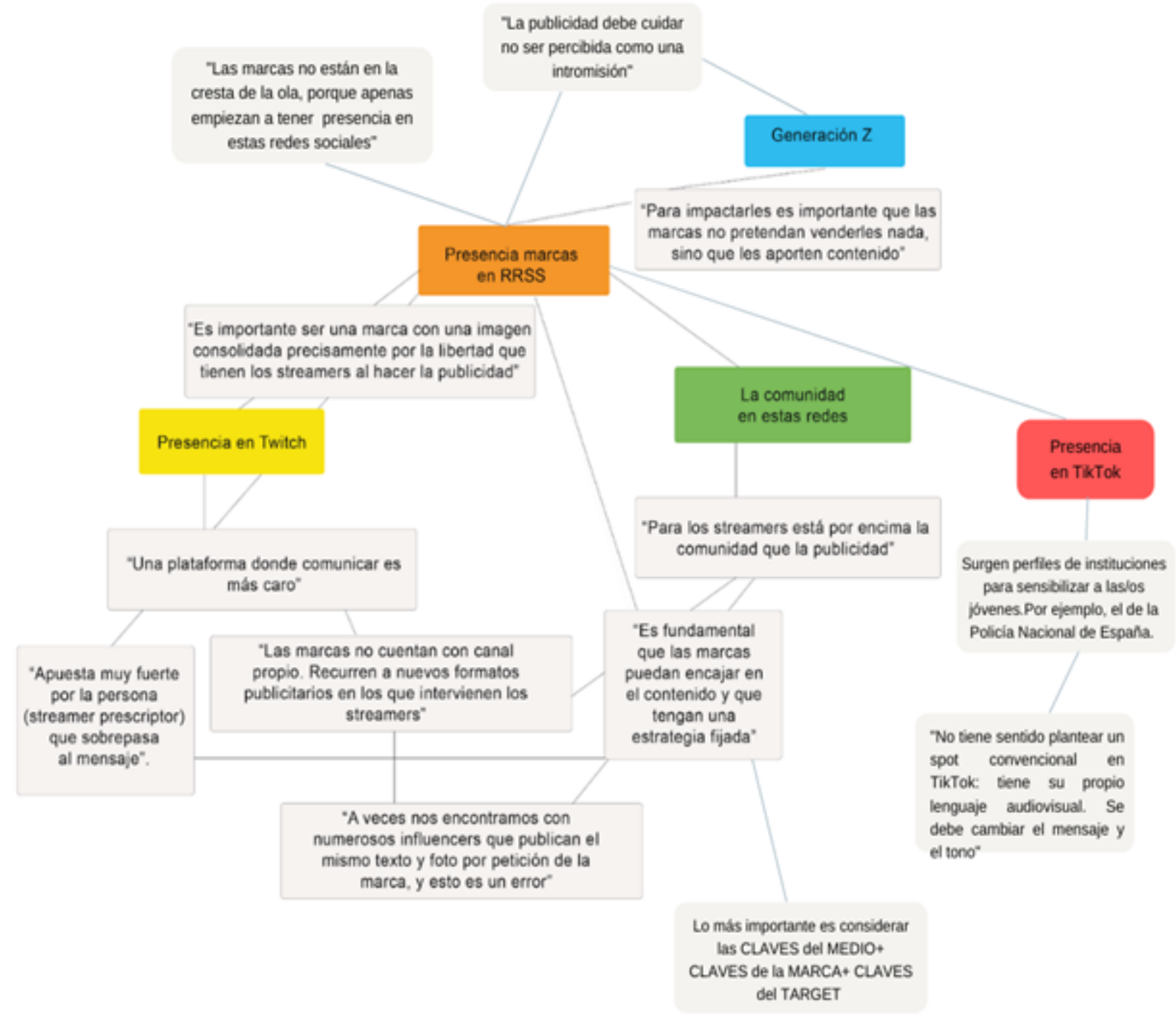

Fuente: Elaboración propia

Los profesionales enfatizan en el hecho de que las estrategias de comunicación de marca han evolucionado, porque en la actualidad es prioritario tener al usuario como elemento clave en cualquier estrategia; pasando el mensaje de la marca a un segundo plano. Además, también hacen referencia a la necesidad de tener una estrategia claramente definida y en la adaptación de mensajes, formatos y tonos a cada canal de comunicación. Al respecto, Magaña (2021) señala que Twitch es "un espacio de oportunidad para las marcas, aunque no todas tengan que estar en todos los sitios porque sí"; esta declaración refleja a la perfección la opinión trasladada por los expertos entrevistados: antes de comunicar en estas plataformas, hay que plantearse si la marca (o su propuesta) encaja en esta plataforma y si va a ser beneficioso para ella y para su público. 
Por otro lado, uno de los aspectos más relevantes de la comunicación en estas redes sociales, especialmente en Twitch, es el hecho de que las marcas pierdan el control del mensaje. Tal y como mencionó Katty Huerta en la entrevista, en plataformas como Instagram las marcas determinan el tipo de contenido que se puede publicar a los influencers. Esto supone un cambio en el paradigma publicitario, ya que actualmente se otorga mayor importancia al prescriptor que al mensaje, y esto genera más libertad y espontaneidad en los mensajes comerciales en Twitch.

Para obtener una mejor comprensión de la información relacionada con las estrategias publicitarias en Twitch, en el Gráfico 3 se exponen las debilidades y oportunidades que brinda esta plataforma, de acuerdo con los expertos entrevistados.

\section{Gráfico 3. Publicidad en Twitch según los expertos}

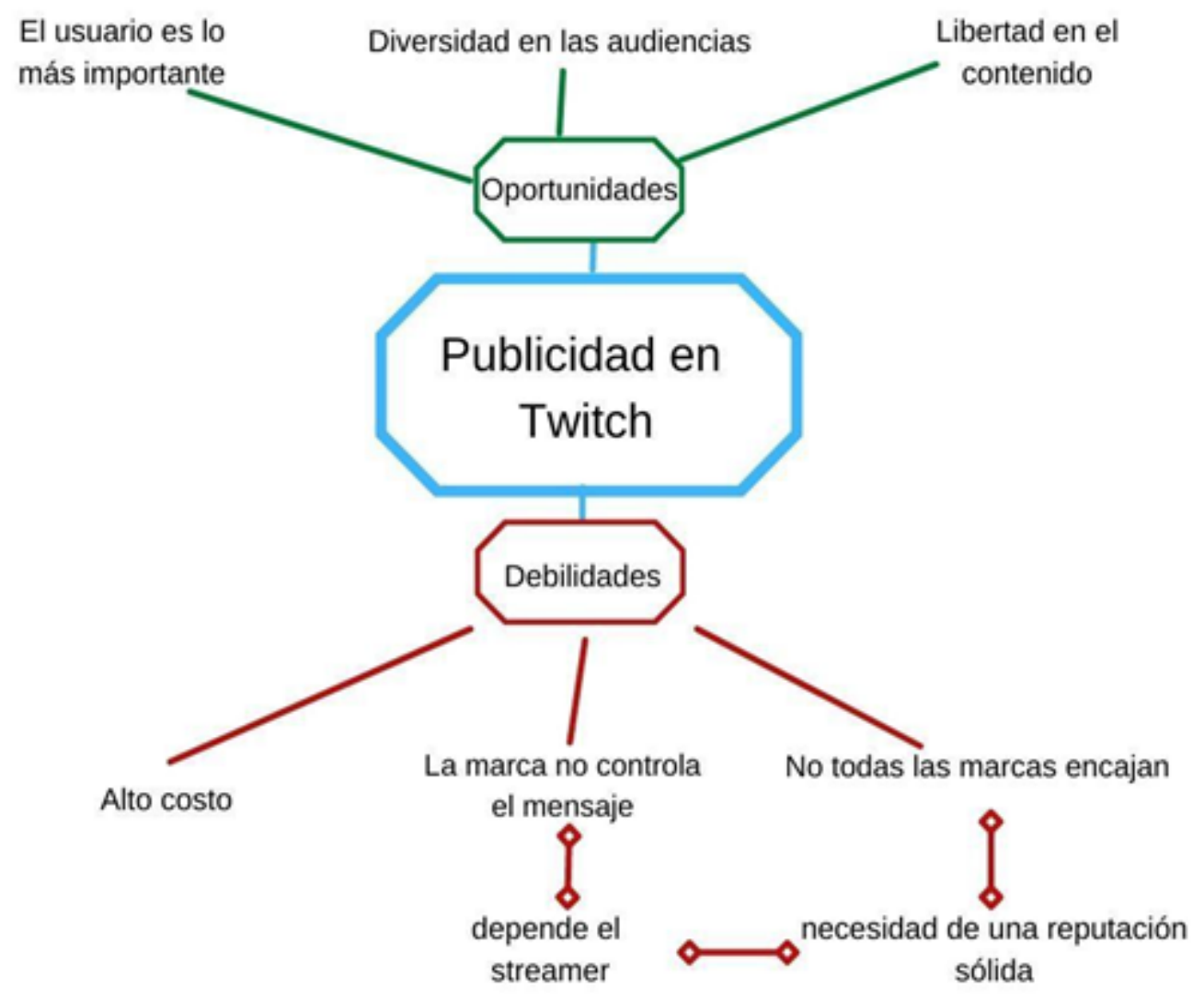

Fuente: Elaboración propia 


\subsection{Encuesta exploratoria}

La encuesta online recibió 420 respuestas de hombres y mujeres de entre 14 y 21 años. Se evidencia que los adolescentes utilizan más TikTok que Twitch: el $63 \%$ de los jóvenes afirma usar TikTok, mientras que el 34\% prefiere Twitch. No obstante, si los datos se segmentan por sexo existen diferencias notables sobre el consumo de ambas plataformas. Respecto a Twitch, el $18,7 \%$ de las mujeres de esta generación asegura utilizarlo, frente al $62 \%$ de los hombres. Sin embargo, en TikTok ocurre lo contrario, siendo más las mujeres usuarias (68\%) que hombres (54\%).

\subsubsection{Consumo de contenidos en Twitch y TikTok}

En cuanto a la tipología de contenidos que se consumen resultan diferencias entre sexo. La aplicación de la prueba de chi-cuadrado determina que no existe dependencia en ninguna de las plataformas entre el sexo y el tipo de contenido consumido

Para TikTok, el valor de chi-cuadrado encontrado fue de $x^{2}=38,741, p=>, 001$. Asumiendo un grado de libertad de (5) y un valor crítico de $x^{2}=11,070$ para ambas plataformas. 
Tabla 2. Consumo de TikTok por tipo de video en la Generación Z

\begin{tabular}{|l|r|r|r|}
\hline \multicolumn{1}{|c|}{ Tipo de contenido } & Mujeres & Hombres & Totales \\
\hline Tips de estudio, decoración y cocina & 44 & 23 & 67 \\
\hline Rutinas diarias & 58 & 15 & 73 \\
\hline Videos cómicos & 37 & 55 & 92 \\
\hline Challenges & 65 & 20 & 85 \\
\hline Material de marcas e influencers & 72 & 30 & 102 \\
\hline Noticias, temas polémicos & 1 & 0 & 1 \\
\hline TOTALES & 277 & 143 & 420 \\
\hline TOTALES\% & $66 \%$ & $34 \%$ & $100 \%$ \\
\hline
\end{tabular}

Fuente: Elaboración propia

En Twitch, el valor de chi-cuadrado encontrado fue de $x^{2}=15,647, p=>, 01$. Por tanto, el tipo de contenido consumido en Twitch no está asociado directamente con el sexo del usuario, por lo que los resultados no se pueden escalar a una conclusión generalizada. 
Tabla 3. Consumo de Twitch por tipo de video en la Generación Z

\begin{tabular}{|l|r|r|r|}
\hline \multicolumn{1}{|c|}{ Tipo de contenido } & Mujeres & Hombres & Totales \\
\hline Partidas de videojuegos & 19 & 74 & 93 \\
\hline Eventos deportivos & 4 & 28 & 32 \\
\hline Entrevistas & 21 & 39 & 60 \\
\hline Eventos musicales & 11 & 14 & 25 \\
\hline Reacciones de vídeos de YouTube & 19 & 47 & 66 \\
\hline Canal de lbai & 22 & 57 & 79 \\
\hline Grupos de estudio & 4 & 2 & 6 \\
\hline TOTALES & 100 & 261 & 361 \\
\hline TOTALES\% & $27,7 \%$ & $72,3 \%$ & $100 \%$ \\
\hline
\end{tabular}

Fuente: Elaboración propia

Además, es importante mencionar la importancia que cobra la sección "para ti" en TikTok. Con la encuesta se confirma que el $95 \%$ de los jóvenes consumen el contenido que aparece en esta sección por encima de los vídeos compartidos por gente a la que siguen.

En cuanto a los resultados de Twitch, la Generación Z tiene una clara preferencia sobre el contenido relacionado con videojuegos y por el canal de Twitch de Ibai, uno de los streamers más reconocidos y consumidos en España. El tercer tipo de contenido favorito son las reacciones a vídeos de YouTube, lo que confirma que Twitch y YouTube son plataformas interconectadas. Por tanto, la elección del ranking de marcas de YouTube para aplicarlo a Twitch al no haber un ranking propio de esta red social ha sido acertada. 
Gráfico 4. Tipos de vídeo consumidos en Twitch

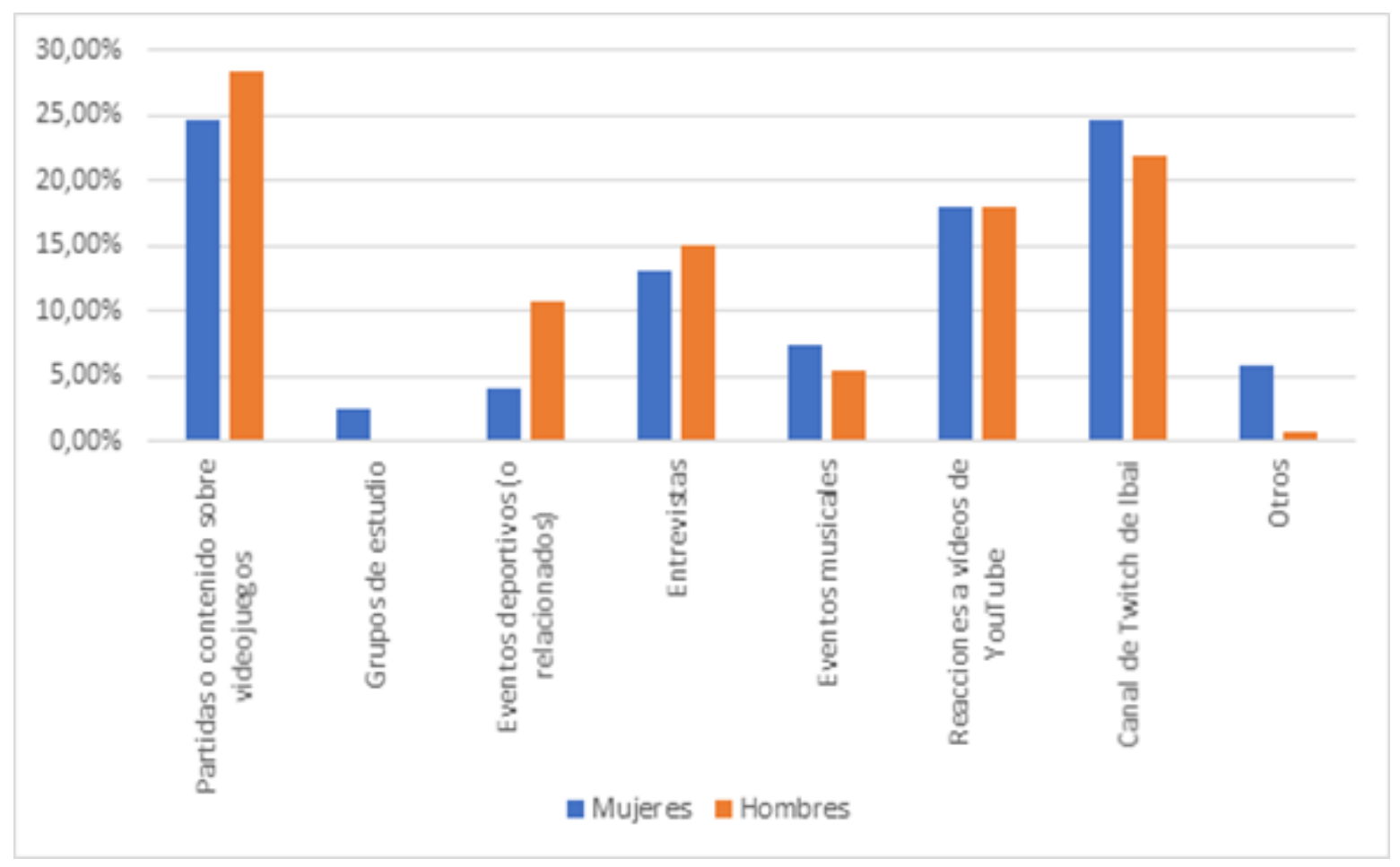

Fuente: Elaboración propia

Otro hallazgo sucede en el tipo de contenido que se consume. Por ejemplo, as mujeres aseguran buscar vídeos de grupos de estudio (un aspecto que ya se había mencionado en el focus group) y en menor medida, vídeos de just chatting o de ASMR (Autonomous Sensory Meridian Response).

\subsubsection{Valoración y reconocimiento de marcas}

En cuanto a la valoración Likert de las marcas en ambas redes sociales, se encuentra que la comunicación mejor valorada de las marcas del ranking de Twitch es la de Red Bull, ya que es la que más número de encuestados considera "buena" y la que menos respuestas de "mala" ha acumulado. Frente a esto, se aprecia que la marca AS tiene el mayor porcentaje de consideración "mala" respecto a todas las marcas, así como el mayor porcentaje de "NS/NC", lo que puede significar que la propuesta de comunicación de esta marca no llega suficientemente a los jóvenes $y$, por tanto, no recuerdan sus mensajes para poder valorarlos.

Teniendo en consideración las respuestas de los hombres, las marcas más valoradas serían Red Bull (coincidiendo con los resultados generales) y LaLiga; mientras que para 
las mujeres serían Red Bull y Telecinco. Un aspecto interesante es que mientras que para las mujeres Telecinco es la segunda marca mejor valorada, para los hombres es la peor valorada de todas. Las respuestas de NS/NC denotan un gran sesgo de sexo en las respuestas, puesto que la menos reconocida por los hombres es Cosmopolitan y, en el caso de las mujeres, las menos reconocidas corresponden a los tres periódicos deportivos incluidos en el listado y al programa de actualidad deportiva El Chiringuito de Jugones.

Las diferencias de valoración de marcas se encuentran señaladas en rojo, naranja y verde en los Gráficos 5 y 6, se relacionan de la siguiente manera: color rojo: mala consideración; color naranja: NS/NC; y color verde: buena consideración.

Gráfico 5. Valoración de marcas en TikTok. Visión masculina

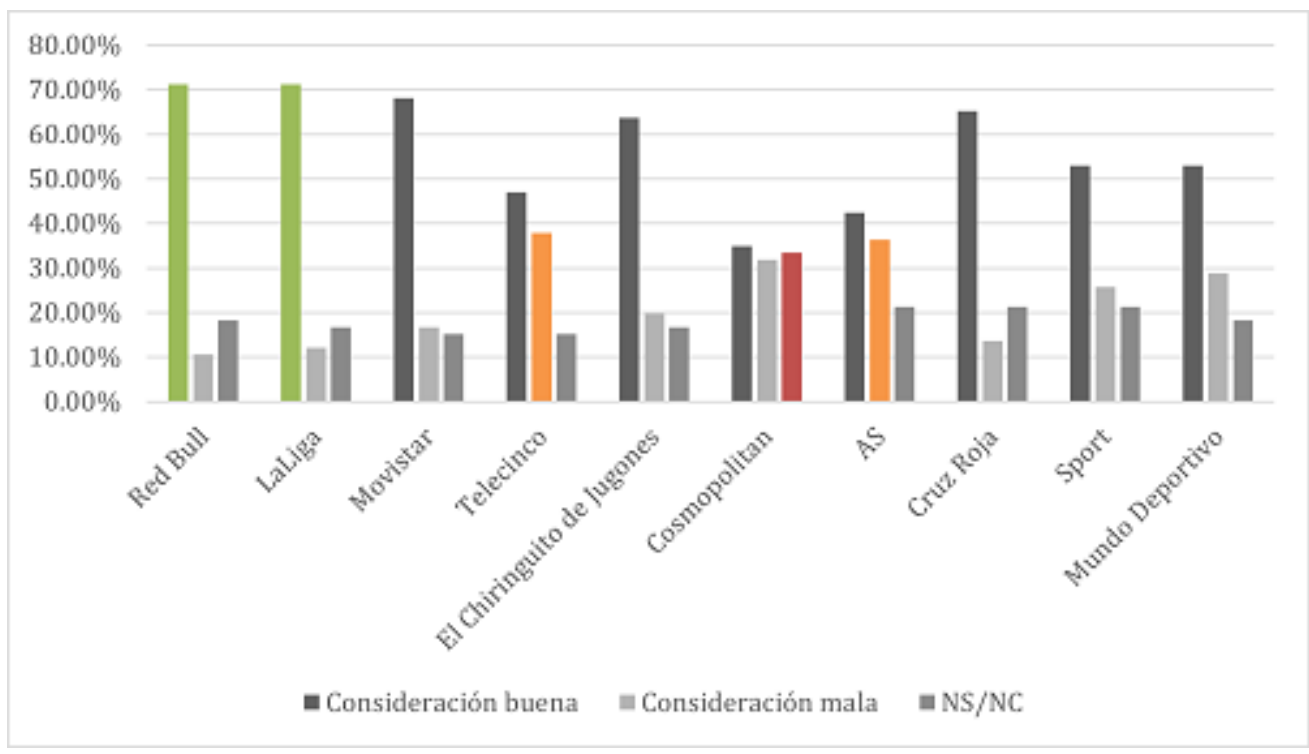

Fuente: Elaboración propia 
Gráfico 6. Valoración de marcas en TikTok. Visión femenina

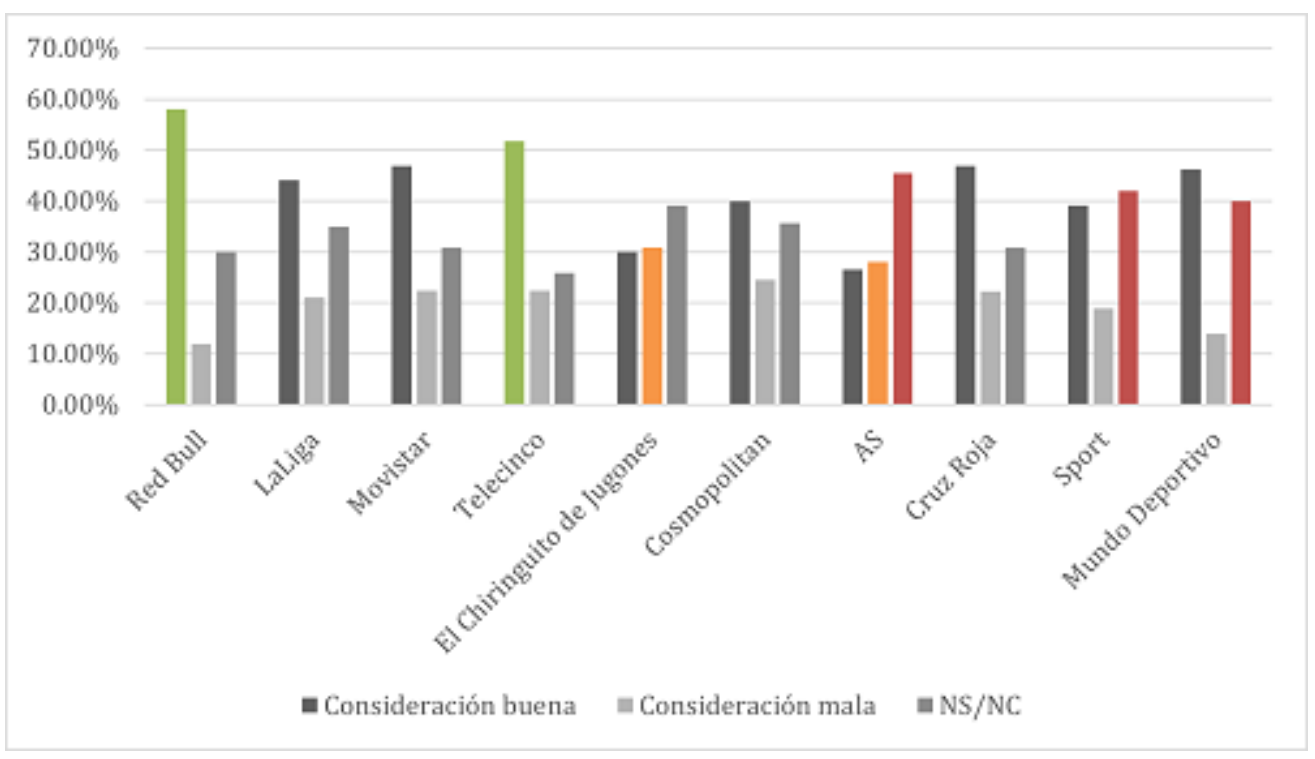

Fuente: Elaboración propia

En cuanto a las marcas que más recuerdan los usuarios, se exigió una respuesta espontánea. Para la evaluación de TikTok, las marcas con mayor presencia pagada no coinciden con las marcas más recordadas por los usuarios y de nuevo hay diferencias entre hombres y mujeres. 
Tabla 4. Reconocimiento de marcas en TikTok

\begin{tabular}{|c|c|c|}
\hline RANKING HOMBRES & RANKING MUJERES & RANKING TOTAL \\
\hline Adidas & Shein & Shein \\
\hline Nike & Nike & Nike \\
\hline Donettes & Maybelline & Adidas \\
\hline Shein & Adidas & Donettes \\
\hline Coca-Cola & Donettes & Maybelline \\
\hline
\end{tabular}

Fuente: Elaboración propia

Las marcas vistas en Twitch de forma espontánea se pueden ver en la Tabla 5. A diferencia con TikTok, tres de las cinco primeras marcas coinciden con las del ranking utilizado para la valoración de las marcas. Esto significa que las marcas con mayor presencia en la plataforma también son las más recordadas y reconocidas; por tanto, realizan un buen ejercicio de comunicación. 
Tabla 5. Reconocimiento de marcas en Twitch

\begin{tabular}{|c|c|c|}
\hline RANKING HOMBRES & RANKING MUJERES & RANKING TOTAL \\
\hline Amazon & Red Bull & Amazon \\
\hline Cola-Cao & Netflix & PlayStation \\
\hline Fornite & PlayStation & Cola-Cao \\
\hline Nike & Domino's Pizza & Red Bull \\
\hline PlayStation & Doritos & Fornite \\
\hline
\end{tabular}

Fuente: Elaboración propia

En cuanto a la valoración de marcas en Twitch, las compañías mejor valoradas son PlayStation, FIFA y Fornite respectivamente, superando todas el $88 \%$ de valoración. Frente a esto, se encuentra el caso de L'Oreal, BBVA, Myprotein y Vodafone, que son las marcas peor valoradas, porque la opción de "mala" es superior al $25 \%$ en todas ellas. También son las que menos reconocimiento tienen por parte de los jóvenes, especialmente Myprotein.

\subsubsection{Publicidad en TikTok y Twitch}

En relación con el tipo de publicidad que más recuerdan los usuarios, se encontró que el patrocinio de eventos o vídeos es la fórmula que más impacta en la Generación Z en Twitch, pues de esta forma es como aparecen la mayoría de las marcas en esta red social. Los jóvenes también señalan que en numerosas ocasiones ven logos de marcas durante los streams. Otra de las diferencias entre ambas redes sociales es la inserción de productos en los vídeos, una estrategia que se identifica más en TikTok. 
La forma más habitual por la que los jóvenes recuerdan haber visto estas marcas en TikTok es a través de anuncios, seguido por la inserción de un producto concreto en un vídeo y por figurar como marca patrocinadora. Los hombres recuerdan más marcas que aparecen en anuncios y/o en filtros o efectos creados por las compañías. Mientras que las mujeres recuerdan más comparaciones/críticas/evaluaciones de productos. Las encuestadas también señalan la colaboración de marcas con tiktokers como un medio a través del cual reconocían haber visto marcas en TikTok.

\section{Gráfico 7. Formas publicitarias en TikTok}

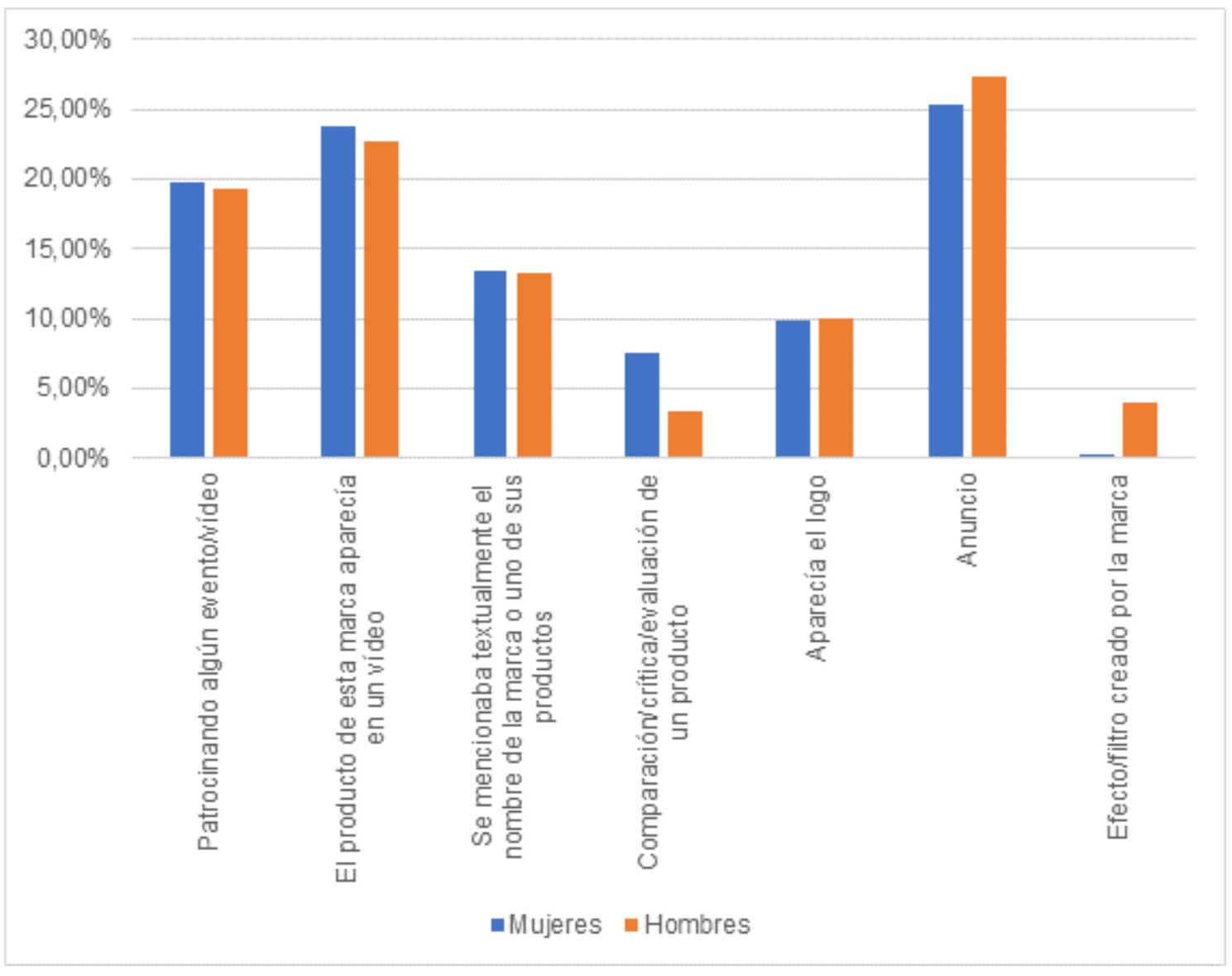

Fuente: Elaboración propia

Una diferencia sobresaliente es que se recuerda más el logo de una marca en Twitch que en TikTok y son las mujeres quienes tienen mayor reconocimiento de los logos en ambas plataformas.

Por otro lado, los hombres utilizan Twitch para conocer o evaluar un producto, mientras que las mujeres lo hacen en TikTok. 
Gráfico 8. Formas publicitarias en TikTok y Twitch. Visión femenina

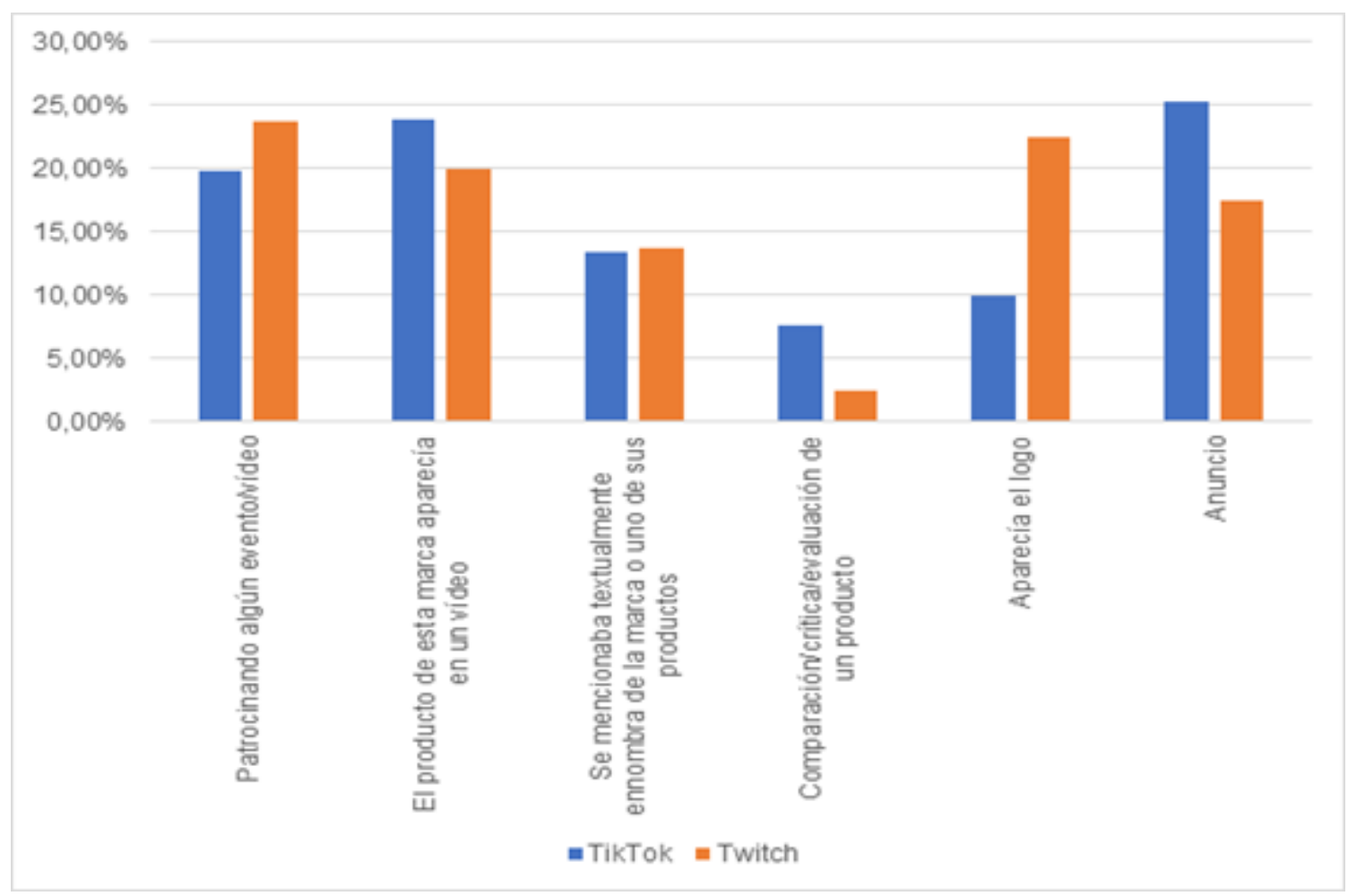

Fuente: Elaboración propia 
Gráfico 9. Formas publicitarias en TikTok y Twitch. Visión masculina

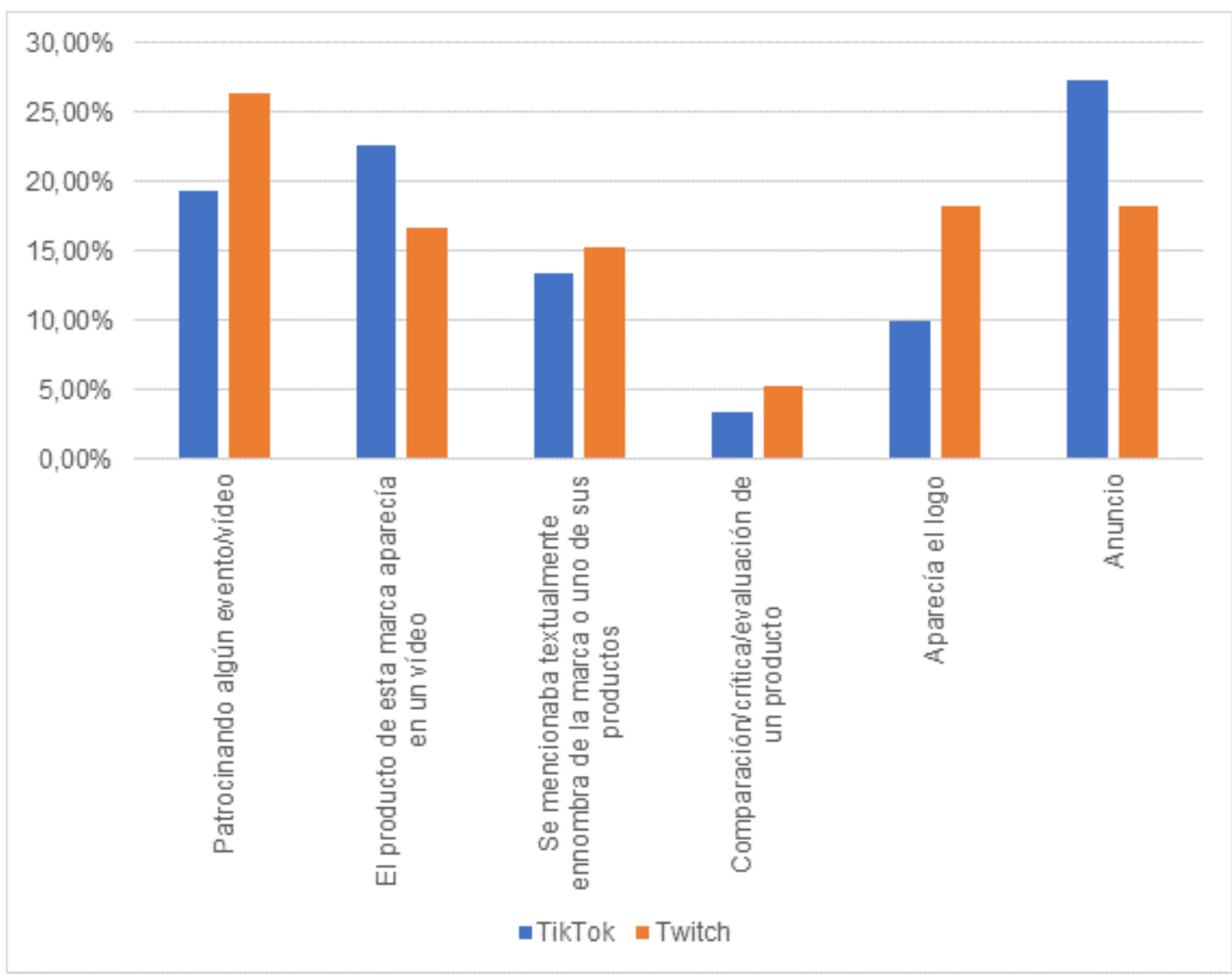

Fuente: Elaboración propia

Por último, a los encuestados se les preguntó sobre cómo les gustaría que fuese la publicidad de las marcas en estas redes sociales y sus respuestas quedan plasmadas en el Gráfico 10. Los usuarios enfatizaron que lo más importante para que la comunicación de las marcas no les resulte molesta es que sea breve, en tono de humor $y$, especialmente, que puedan ser capaces de evitar esta publicidad. Además, les interesa que las marcas ofrezcan algún tipo de recompensa, como es el caso de códigos de regalo. 
Gráfico 10. Publicidad ideal en TikTok y Twitch según la Generación Z

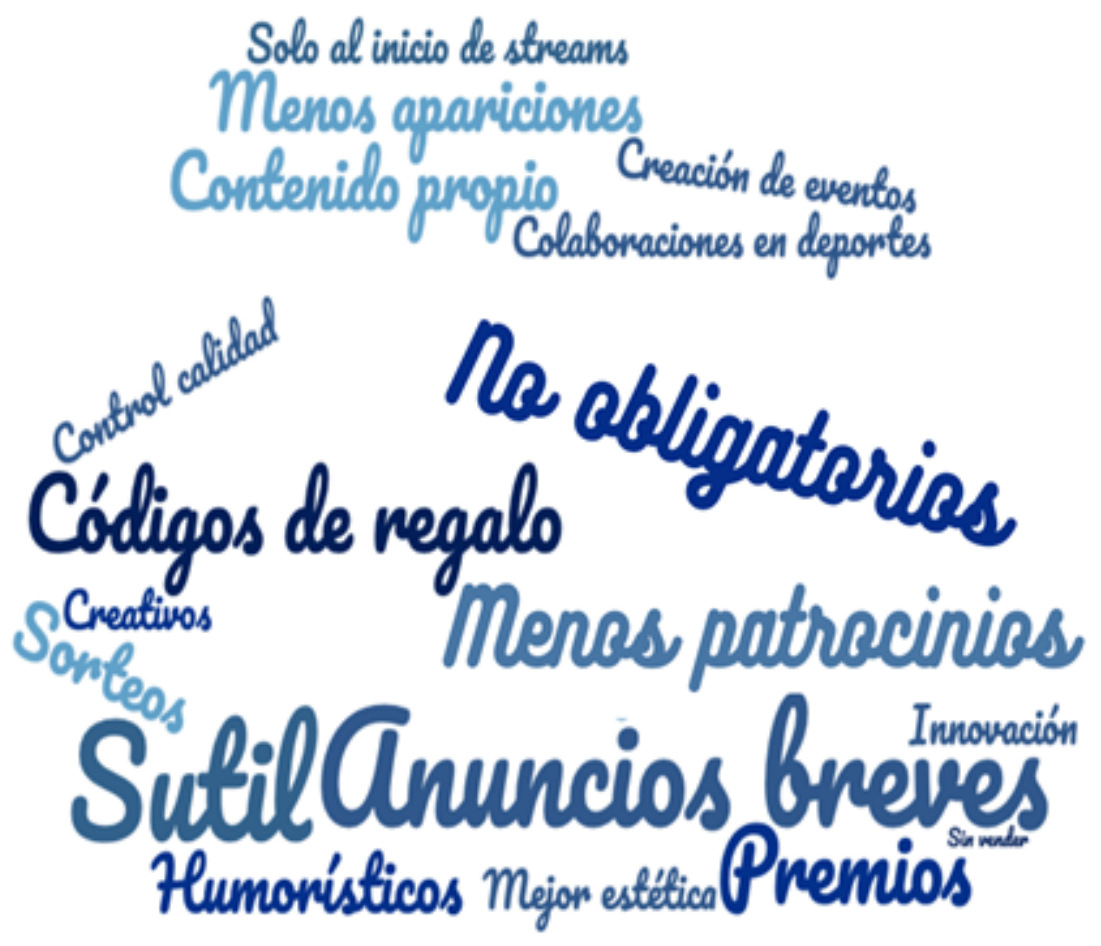

Fuente: Elaboración propia

\section{Discusión y Conclusiones}

De acuerdo con la información recabada, es innegable que en el año 2020 las plataformas como TikTok y Twitch se convirtieron en tendencia digital global, ocasionando que las marcas se interesen por adaptar sus contenidos a estas redes. También, queda claro que las marcas están interesadas en llegar a un público más diverso y para ello deben cambiar sus estrategias de comunicación.

En el caso específico de la Generación Z, como mencionan Martín y Medina (2021), las redes sociales se presentan como su principal medio de comunicación y un lugar donde pasan gran parte del tiempo. Los usuarios afirman que pasan más de tres horas diarias entre TikTok y Twitch, aunque se debe resaltar que si en Twitch existe algún evento en directo que sea de su interés pueden estar hasta seis horas consecutivas. Además de ser un medio para obtener entretenimiento, el consumo de estas redes sociales también se ha transformado. De hecho, la mitad de los encuestados aseguran que utilizan estas plataformas para estudiar en grupo y para seguir consejos de estudio, cocina y decoración, particularmente en TikTok. En cuanto a los vídeos de ASMR de Twitch, estos tienen mucho que ver con el uso de estas plataformas con fines educativos. Son vídeos en los que 
el sonido es el elemento principal, pasando la imagen a un segundo plano. En ellos, el streamer retransmite ciertos patrones sonoros para inducir relajación y tranquilidad a los espectadores.

El hecho de que miembros de la Generación Z estén interesados en este tipo de contenido pone en evidencia que los jóvenes no sólo buscan entretenimiento en estas plataformas, sino que quieren encontrar herramientas para mejorar su rendimiento en los estudios, cuidar su salud mental, o para su día a día (cómo ya se evidenciaba en los resultados de TikTok al ver que el segundo tipo de video preferido eran los tips de estudio, decoración o cocina). Vinculado con esto, es importante recordar que, tal y como se pudo recoger del focus group llevado a cabo, la Generación Z no es consumidora de contenidos producidos por miembros de su misma generación, a no ser que sean sus amigos. Renés, Gozálvez y Berlanga (2020) ya hacen referencia a ello en su trabajo.

En este sentido, tanto expertos como usuarios, coinciden en que las publicaciones más exitosas y seguidas en TikTok no son únicamente humorísticas, también hay interés en seguir diarios de vida. Estos relatos de la vida cotidiana de alguien suponen una oportunidad para las marcas, pues pueden tener presencia en estos vídeos de forma sutil para llegar a los jóvenes, porque de acuerdo con los resultados de esta investigación, ellos sí recuerdan y reconocen las marcas que aparecen.

A pesar de que TikTok sí ofrece la posibilidad de que las marcas tengan una cuenta propia, los usuarios afirmaron que no suelen seguir estos perfiles y solo suelen consumir los vídeos que aparecen en la sección "para ti". Esto desemboca en la necesidad de que las marcas elijan correctamente los hashtags de sus publicaciones para que estas aparezcan asertivamente en la sección "para tí" de su público objetivo.

En el caso de Twitch se debe remarcar que, al no contar con canal propio de Twitch, las marcas no tienen el control absoluto de sus mensajes al depender del prescriptor que mencione/utilice su producto. Por esta razón, es necesario contar con una imagen y reputación sólidas que no puedan verse mermadas por estas colaboraciones incontroladas. Esto requiere una planificación offline y online.

Mir (2016) y Costa (2013) puntualizan que las empresas necesitan estar presentes en redes sociales para no perder la interacción con sus públicos. Sin embargo, al tratarse de dos plataformas que son tan cambiantes y pueden llegar a ser efímeras, el elemento más importante para las marcas no debe de ser el mensaje comercial sino el usuario: se hace necesario poner el foco sobre el target antes que en el discurso publicitario. La marca no debe ser la protagonista.

Asimismo, resulta indispensable que las compañías sean conscientes de que no pueden pretender vender sus productos a través de anuncios, sino que deben evolucionar en su discurso y centrarse en crear contenido atractivo para su público, por ejemplo, participando en challenges que sean tendencia. 
Por supuesto, las marcas también deben saber adaptar su mensaje a las diferentes plataformas y ofrecer contenido de calidad acorde al medio en el que están presentes, aunque los expertos recalcan que no necesariamente las marcas deben estar en todos los canales si no es necesario.

Adicionalmente, de acuerdo con los resultados obtenidos, los usuarios exigen que los anuncios sean más creativos, breves y que les brinden algún tipo de beneficio. La publicidad convencional pagada llega a ser molesta para los jóvenes, ocasionando un rechazo posterior a esa marca, un aspecto que también afirma Ferrer (2020).

Los miembros de la Generación Z valoran mejor la comunicación de las marcas Twitch que en TikTok, a pesar de ser la menos utilizada de las dos y la que representa mayor inversión para las marcas. Por tanto, se puede concluir que una aparición en un stream de Twitch resulta más eficaz que tener presencia en TikTok. Pollack et al. (2021) ya mencionaban la eficacia de la publicidad en Twitch frente a las plataformas asíncronas, como YouTube. Las acciones en Twitch aumentan el reconocimiento de marca, a pesar de que la marca no sea del interés del usuario. Del mismo modo, el poco reconocimiento de las marcas se puede relacionar con una baja de credibilidad de estas.

En conclusión, las marcas necesitan establecer estrategias específicas para cada red, y ser conscientes de que no todos los productos encajan en todas las plataformas y en todos los tipos de vídeos o streams. La Generación Z busca contenidos propios, innovadores y que no tengan como finalidad única vender. Para ellos interactuar con las marcas en estas plataformas no resulta importante, por eso demandan contenidos sobre temas de actualidad y que estén relacionados con sus intereses personales: este contenido de valor ayudará a fidelizar a los usuarios jóvenes y a conseguir que sean consumidores futuros de estas marcas.

\section{Referencias bibliográficas}

2tube. (2020). Marcas. http://2btube.com/marcas/

Álvarez, E., Heredia, H. y Romero, M.F. (2019). La Generación Z y las Redes Sociales. Una visión desde los adolescentes en España. Revista Espacios, 40(20), 1-13.

Batthyány, K. y Cabrera, M. (2011). Metodología de la investigación en Ciencias Sociales. Apuntes para un curso inicial. Montevideo: Universidad de la República.

Castelló-Martínez, A. (2020). Análisis interdisciplinar de la serie Élite (Netflix): narrativas transmedia, generación Z, tendencias del consumidor y brand placement. Revista Inclusiones, 7, 1-26.

Castelló Martínez, A., y Del Pino Romero, C. (2015). La comunicación publicitaria con Influencers. Redmarka. Revista De Marketing Aplicado, 14, 21-50. https://doi.org/10.17979/redma.2015.01. 014.4880

Chaoudhary, N., Gautam, C. y Arya, V. (2020). Digital marketing challenge and opportunity with reference to TikTok a new rising social media platform. International Journal of Multidisciplinary Educational Research, 9(10), 189-197. 
Cooley, D., y Parks-Yancy, R. (2019). The Effect of Social Media on Perceived Information Credibility and Decision Making. Journal of Internet Commerce, 18(3), 249-269. https://doi.org/10.1080/15 332861.2019 .1595362

Costa, J. (2013). Los 5 pilares del branding. Barcelona: CPC Editor.

Elías, M. (2021). Deseos alcanzables. Blog de Collabora Brands. https://bit.ly/32TfexQ

Epsilon Technologies. (2020). Tendencias y Rankings de top marcas en TikTok España. https://bit.ly/3 aKxWMq

Espinar, E., Zych, I., y Rodríguez-Hidalgo, A. J. (2015). Ciberconducta y dependencia emocional en parejas jóvenes. Psychology, Society, \& Education, 7(1), 41-55.

Fernández de la Iglesia, J.C., Casal, L., Fernández, M.C. y Cebreiro, B. (2020). Actitudes y uso de Internet y redes sociales en estudiantes universitarios/as de Galicia: implicaciones personales y sociales. Revista Prisma Social, 28, 145-160. https://revistaprismasocial.es/article/view/3372

Ferrer, M. (2020). Neuromarketing y la medición del efecto de la publicidad de influencers en adolescentes. Revista Mediterránea de Comunicación, 11(2), 241-259. https://doi.org/10.14198/ MEDCOM2020.11.2.11

Francisco, N. y Rodríguez, A.I. (2020). La innovación de la Televisión Pública Europea en la oferta audiovisual digital: nuevas plataformas para la Generación Z. Revista de la Asociación Española de Investigación de la Comunicación, 7(13), 185-212. https://doi.org/10.24137/raeic.7.13.9

Gálvez, M. (2017). La publicidad digital en manos de los usuarios: más allá del ad-blocking. Madrid: Publicis Media y AEDEMO. https://bit.ly/2ECPTUu

García-Jiménez, A., Tur-Viñes, V., y Pastor-Ruiz, Y. (2018). Consumo mediático de adolescentes y jóvenes. Noticias, contenidos audiovisuales y medición de audiencias. Icono 14, 16(1), 22-46. $\underline{\mathrm{h}}$ ttps://doi.org/10.7195/ri14.v16i1.1101

Gutiérrez, J.F. y Cuartero, A. (2020). El auge de Twitch: nuevas ofertas audiovisuales y cambios del consumo televisivo entre la audiencia juvenil. Ámbitos: Revista Internacional de Comunicación, 50, 159-175. https://doi.org/10.12795/Ambitos.2020.i50.11

IAB. (2021). Publicidad digital 2021. shorturl.at/julZ9

Jung, H., y Phoa, F. K. H. (2021). On the effects of capability and popularity on network dynamics with applications to YouTube and Twitch networks. Physica A: Statistical Mechanics and its Applications, 571, 125663. https://doi.org/10.1016/j.physa.2020.125663

Karbaum, G. (2018). Narrativas social media y el prosumidor mediático. Correspondencias \& Análisis, 8, 219-238. doi: https://doi.org/10.24265/cian.2018.n8.11

Keller, K. y Swaminathan, V. (2019). Strategic Brand Management: Building, Measuring, and Managing Brand Equity. Londres: Pearson Education.

Lacasa, P. (2018). Expresiones del futuro. Cómo se comunicarán las próximas generaciones. Madrid: Morata.

Li, X., Xiaohui, Y. y Zhengwu, Z. (2019). Research on the causes of the «TikTok» app becoming popular and the existing problems. Journal of advanced management science, 7(2), 59-63. https://doi.o rg/10.18178/joams.7.2.59-63

Magaña, R. (2021). Twitch y el valor de la naturalidad. ¿Es Twitch la televisión de la Generación Z? Reason Why. https://bit.ly/3gIVYLu

Marcos, M., Pérez, M.P.M., Cerezo, M. y Hernández, M. (2020). Infancia y contenidos audiovisuales online en España: una aproximación al consumo y a la mediación parental en las plataformas OTT. Icono 14. Revista Científica De Comunicación Y Tecnologías Emergentes, 18(2), 245-268. http s://doi.org/10.7195/ri14.v18i2.1560 
Marinas, L. (2019). Instagram: Donde Millennials, Generación Z, McLuhan y Bolter se cruzan. Cuadernos de la Información y Comunicación, 24, 187-201. https://doi.org/10.5209/ciyc.64641

Martín, D. y Medina, M. (2021). Redes sociales y la adicción al like de la generación Z. Revista de Comunicación y Salud, 11, 55-76. https://doi.org/10.35669/rcys.2021.11.e281

Martínez, E., García, A. y Sendín, J. C. (2013). Percepción de los riesgos en la red por los adolescentes en España: Usos problemáticos y formas de control. Anàlisi: Quaderns De Comunicació i Cultura, 48, 111-130. http://dx.doi.org.bucm.idm.oclc.org/10.7238/a.v0iM.1969

Martínez-Costa, M.P., Serrano-Puche, J., Portilla, I., Sánchez-Blanco, C. (2019). La interacción de los jóvenes adultos con las noticias y la publicidad online. Comunicar, 59(27), 19-28. https://doi.o rg/10.3916/C59-2019-02.

Mir, P. (2016). Brand.com: reputación de marcas y social media. Pamplona: Eunsa.

Navarro, M. y Vázquez, T. (2020). El consumo audiovisual de la Generación Z. El predominio del vídeo online sobre la televisión tradicional. Ámbitos: Revista Internacional de Comunicación, 51, 10-30. https://doi.org/10.12795/Ambitos.2020.i50.02

Pedroni, M. (2013). The Crossroad between Production and Consumption: An Introduction to Fashion as a Cultural Industry. En M.L., Pedroni (Ed.), From production to consumption: The cultural industry of fashion (pp. VII- XLVII). Oxford, UK: Inter-Disciplinary Press.

Pires, K., y Simon, G. (2015, March). YouTube live and Twitch: a tour of user-generated live streaming systems. In Proceedings of the 6th ACM multimedia systems conference (pp. 225-230). https://do i.org/10.1145/2713168.2713195

Pollack, C., Gilbert-Diamond, D., Emond, J., Eschholz, A., Evans, R., Boyland, E., y Masterson, T. (2021). Twitch user perceptions, attitudes and behaviours in relation to food and beverage marketing on Twitch compared with YouTube. Journal of Nutritional Science, 10, E32. https://doi:10.1017 /jns.2021.22

Renés Arellano, P., Gozálvez Pérez, V., y Berlanga Fernández, I. (2020). YouTube e influencers en la infancia. Análisis de contenidos y propuestas educativas. Icono 14. Revista Científica De Comunicación Y Tecnologías Emergentes, 18(2), 269-295. https://doi.org/10.7195/ri14.v18i2.1455

Scully-Blaker, R., Begy, J., Consalvo, M., y Ganzon, S. (2017). Playing along and playing for on Twitch: Livestreaming from tandem play to performance. Proceedings of the 50th Hawaii International Conference on System Sciences.

Segovia, B., Mérida, R., Olivares, M.A. y González, E. (2016). Procesos de socialización con redes sociales en la adolescencia. Revista Latinoamericana de Tecnología Educativa, 15(3), 155-167. ht tp://dx.medra.org/10.17398/1695-288X.15.3.155

Shuai, Y., Yuzhen, Z. y Yifang, M. (2019). Analysis of the reasons and development of short video application Taking Tik Tok as an example. 2019 9th International Conference on Information and Social Science (ICISS 2019) (pp. 340-343). Francis Academic Press.

Sidhu, P., y Carter, M. (2020). The critical role of YouTube and Twitch in D\&D's resurgence. Proceedings of DiGRA Australia 2020, 1-3.

Soler, A.M. (2016). La confianza de los adolescentes escolarizados en las redes sociales. Praxis \& Saber, 7(15), 231-246. https://doi.org/10.19053/22160159.v7.n15.2016.5734

Suárez-Álvarez, R. y García-Jiménez, A. (2021). Centennials en TikTok: tipología de vídeos. Análisis y comparativa España-Gran Bretaña por género, edad y nacionalidad. Revista Latina de Comunicación Social, 79, 1-22. https://www.doi.org/10.4185/RLCS-2021-1503

Thomas, M. (2020, 19 de noviembre). 5 Gen Z marketing strategies to TikTok (2021). TikTok for business. shorturl.at/rIX04 
Toffler, A. (1980). The Third Wave. William Morrow.

Tur-Viñes, V., Núñez-Gómez, P., y González-Río, M.J. (2018). Menores influyentes en YouTube. Un espacio para la responsabilidad. Revista Latina de Comunicación Social, 73, 1211-1230. doi:10.4185/RLCS-2018-1303

Twitch (2021). Informe de transparencia 2020. shorturl.at/mCI79

Varona, D. (2013). Canal Clan de RTVE: la iniciación de los niños en las redes. En Ron, R., Álvarez, A. y Núnez, P. Niños, adolescentes y redes sociales. ¿Conectados o atrapados? ESIC.

Vilanova, N. y Ortega, I. (2017). Generación Z: Todo lo que necesitas saber sobre los jóvenes que han dejado viejos a los millennials. Barcelona: Plataforma Editorial.

Wilson, S. (2021, 11 de marzo). Where brands are reaching Gen Z. Harvard Business Review. https:/ /hbr.org/2021/03/where-brands-are-reaching-gen-z\# 